\title{
UVAS: FORMAS DE USO, CONSUMO E ATIVIDADES BIOLÓGICAS - UMA REVISÃO
}

\section{GRAPES: THE FORMS OF USE, CONSUMPTION AND BIOLOGICAL ACTIVITIES - A REVIEW}

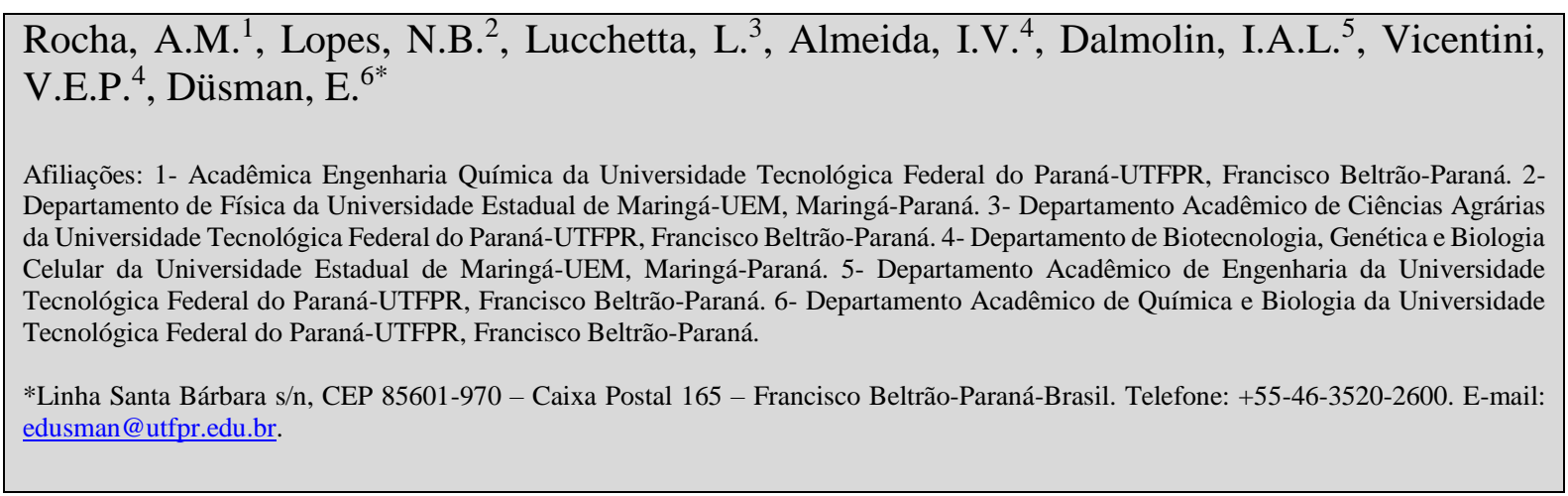

\section{Resumo}

A vitivinicultura é uma atividade economicamente importante no mundo globalizado, gerando emprego, renda e investimentos. As uvas são consideradas como uma das maiores fontes de compostos fenólicos, contendo ainda compostos flavonoides e não-flavonoides na sua composição, a se destacar o resveratrol. $O$ consumo das uvas pode ser in natura, ou na forma de derivados como vinhos, sucos, geleias, dentre outros. Entretanto, o processamento das uvas gera vários resíduos, como o engaço, a borra e em maior quantidade o bagaço, que é formado principalmente de cascas e sementes. Da mesma forma que existem compostos benéficos na polpa da fruta, muitos antioxidantes e outros compostos ainda permanecem nestes resíduos. Assim, dentre as inúmeras propriedades medicinais das uvas e dos extratos produzidos desta ou de seus resíduos, pode-se citar: auxiliar no tratamento de hemorragias, dor de garganta, diarreia, cólera, varíola, náuseas, infecções nos olhos e pele, doenças cardiovasculares, hipertensão, auxílio do tratamento da doença de Alzheimer e Parkinson, dentre outras. Além disso, apresentam atividade citotóxica contra células cancerígenas, efeito quimiopreventivo, proteção contra os radicais livres, a peroxidação lipídica e a danos no ácido desoxirribonucleico, além de atividade antimutagênica. Assim, considerando seus benefícios, é importante a realização de estudos que incentivem ainda mais o uso e consumo de uvas, seus derivados e até mesmo o reaproveitamento dos seus resíduos, pela extração dos antioxidantes e os compostos de interesse, empregando no tratamento de diversas doenças.

Palavras-chave: Antioxidantes; Qualidade de Vida; Saúde.

\section{Abstract}

The vitiviniculture is an economically important activity in the globalized world. This ativicty generates jobs, income and investments. Grapes are considerated as one of the largest sources of phenolic compounds, also containing flavonoid and non-flavonoid compounds in their composition, especially resveratrol. The consumption of grapes can be in natura, or in the form of derivatives such as wines, juices, jellies, among others. However, with the processing of grapes are generate various residues, such as stems, sludge and the largest amount of bagasse, which is mainly formed by peels and seeds. Similary as there are beneficial compounds in fruit, many antioxidants and other compounds still remain in this residues. Thus, among the various medicinal properties of grapes and of its extracts obtained from residues, can be cited: help in the treatment of bleeding, sore throat, diarrhea, cholera, smallpox, nausea, eye and skin lesions, cardiovascular disease, hypertension, aid in the treatment of Alzheimer's and Parkinson's disease, among others. In addition, to present cytotoxic activity against cancer cells, chemopreventive effect, protection against free radicals, lipid peroxidation and deoxyribonucleic acid damage and antimutagenic activity. Thus, considering its benefits, it is important to conduct studies that further encourage the use and consumption of grapes, their derivatives and even the reuse of their residues, through the extraction of antioxidants and compounds of interest, applicating for the treatment of various diseases.

Keywords: Antioxidants; Quality of life; Health. 


\section{Introdução}

A vitivinicultura é uma atividade economicamente importante no mundo. No Brasil, é uma atividade que gera emprego e renda, visto que a maior parte da produção advém de pequenas propriedades relacionadas a agricultura familiar. Além disso, grandes investimentos são realizados por meio de empresas de médio e grande porte na área da produção de uvas de mesa e uvas para o processamento, movimentando a economia ${ }^{1}$.

Embora tenha começado de maneira tímida e com muitas dificuldades, a vitivinicultura brasileira pertence ao chamado novo mundo vitivinícola, do qual fazem parte países como China, Argentina, Estados Unidos, África do Sul, Austrália e outros. Nas últimas décadas houve um grande aumento dessa produção devido a expansão da área cultivada e da melhoria da tecnologia da produção de uvas e da elaboração dos vinhos ${ }^{2}$.

A região Sudoeste do Paraná destaca-se na produção de uva orgânica e derivados, o que comprova a valorização de alimentos produzidos em sistemas que estabeleçam um compromisso com a preservação ambiental, a saúde do produtor e do consumidor. Esta concepção fortalece a estrutura de produção numa consonância com meios naturais e uso de insumos que não interferem de maneira agressiva o meio ambiente, como por exemplo, o uso de produtos à base de cobre, como a calda bordalesa $^{3}$. Isto vai na direção contrária da produção dita convencional, que lança mão do uso de agroquímicos, que por vezes ocorre indiscriminados.

Além disso, acredita-se que a concentração de compostos fenólicos seja maior nas plantas de produção orgânica ${ }^{4-6}$. Neste sentido, vale destacar que as uvas são consideradas como uma das maiores fontes de compostos fenólicos ${ }^{5}$, e estes podem ser classificados em flavonoides (catequina, epicatequina, epigalocatequina, caempferol, quercetina, miricetina e antocianinas) e nãoflavonoides (ácidos fenólicos, hidroxibenzóicos, hidroxicinâmicos e o resveratrol $)^{8-10}$.

Considerando a importância econômica

e as destacadas propriedades nutricionais e funcionais da uva, este trabalho busca apresentar o estado da arte desta fruta em suas formas de consumo, uso e suas atividades biológicas.

\section{Métodos}


O presente estudo trata de uma revisão de literatura realizada por meio de buscas em plataformas de dados como: ScienceDirect, SciElo, Google Acadêmico, Portal da Capes, Academia e BDTD, bem como alguns dados de estudos já realizados pelo grupo de pesquisa dos autores deste trabalho. Utilizou-se os seguintes descritores: uva, vinificação, bagaço de uva, antioxidantes, câncer e atividades citotóxicas nos idiomas correspondentes ao banco de dados.

Um dos critérios de seleção foi artigos publicados nos últimos vinte anos, incluindo ensaios originais, revisões, teses e dissertações.

\section{Desenvolvimento}

As uvas possuem uma grande produção e consumo no Brasil. Comumente, as uvas são consumidas in natura ou seu consumo está associado a produção de diversos alimentos como o vinho, suco da uva, geleias, dentre outros $^{11}$. De acordo com Mello ${ }^{12}$, aproximadamente metade da produção da uva no país é destinada ao processamento para realização de vinhos, suco de uva e derivados.

A produção e a comercialização do suco de uva brasileiro têm aumentando anualmente, principalmente, devido às características organolépticas (cor, odor e sabor) e seu valor nutricional, sendo uma alternativa economicamente viável e sustentável aos pequenos agricultores ${ }^{13,14}$. De acordo com a União Brasileira de Viticultura ${ }^{15}$, houve um aumento de $137 \%$ na comercialização de sucos de uva no mercado interno e $27 \%$ no mercado externo, entre o ano de 2008 e 2013.

O suco de uva integral é um líquido límpido ou turvo extraído da uva por meio de processos tecnológicos adequados, apresentando sua concentração e composição natural, sem a adição de açúcar ${ }^{13}$. No Brasil, as uvas do grupo das americanas (Vitis labrusca L.), como as cultivares Concord, Isabel e Bordô, são empregadas na elaboração de sucos pois preservam as características aromáticas e gustativas das uvas no processo de produção e conservação, mesmo quando o suco é aquecido para extração da cor e para pasteurização ${ }^{13}$.

No Paraná, a região Sudoeste responde por $43 \%$ da área plantada de uva e é responsável por $50 \%$ da produção no estado. As cultivares com maior destaque são as Concord, Isabel e Niágara, e algumas cultivares viníferas (Vitis vinifera $\mathrm{L}$.) em menor proporção, como é o caso das cv. Merlot, Cabernet Sauvignon e Moscato ${ }^{16}$. 
A variedade Concord é uma uva bastante utilizada para a elaboração de derivados de uva, principalmente os sucos, devido às suas características organolépticas ${ }^{17}$.

O processamento das uvas gera vários resíduos da vinificação, como o bagaço, o engaço e a borra. $\mathrm{O}$ bagaço é considerado o principal resíduo da vinificação, por ser o de maior volume quando comparado aos outros resíduos gerados, pela sua riqueza alcoólica e tartárica e pelo interesse econômico de alguns compostos. Além disso, é o produto resultante da prensagem das uvas, composto pelas partes sólidas (como as sementes, casca e certa quantidade de engaço) e pelo mosto. O engaço é definido como o conjunto do pedúnculo e ramificações que sustentam o cacho. A borra é a parte sólida formada por precipitantes como floculantes, leveduras e outras substâncias que podem conferir gosto e aroma desagradável ao vinho. O mosto é o sumo da uva que ainda não passou pelo processo de fermentação ${ }^{18}$.

No Brasil, uma pequena quantidade do bagaço é utilizada para a produção de destilado de uva (também chamado de graspa), porém a maior parte é desperdiçada ou utilizada para adubação do solo e complemento de ração animal, já que são fontes de fibras e proteínas.
Com relação à adubação, o seu uso frequente não é conveniente, pois a biodegradação das sementes de uva é lenta, não convertendo toda a matéria orgânica até a chegada de outra safra. Também há uma desvantagem em relação à alimentação dos animais, pois o bagaço não pode ser oferecido puro, devido a sua grande quantidade de fibras, e de tal forma deve ser triturado e misturado com produtos complementares, tornando inviável seu consumo em grande escala ${ }^{19}$.

Da mesma forma que existem compostos benéficos na polpa da fruta, muitos antioxidantes e outros compostos ainda permanecem no bagaço. Por isso a aplicação desses resíduos para a obtenção de compostos bioativos pode representar um ganho econômico e científico ${ }^{18}$.

\section{Discussão}

Dentre as inúmeras propriedades medicinais das uvas e dos extratos produzidos destas ou de seus resíduos, principalmente das cascas e sementes, pode-se citar os potenciais benéficos no tratamento de hemorragias, dor de garganta, diarreia, cólera, varíola, náuseas, 
infecções nos olhos e pele ${ }^{20}$, doenças cardiovasculares $^{9,20-27}$, hipertensão ${ }^{28}$, doenças hepáticas e renais ${ }^{9,20,29-35}$, hiperglicemia ${ }^{36}$, constipação e como diurética ${ }^{20,29}$.

Também foi demonstrado que os extratos de uvas protegem ratos contra a redução da fertilidade 37,38 , além de ter efeitos antienvelhecimento. Pesquisadores também descobriram que camundongos tratados com extrato de sementes de uva reduziram significativamente a deterioração cognitiva da doença de Alzheimer em comparação com os ratos controle ${ }^{39}$. E, que extratos de sementes de uva possuem capacidade quelante de ferro, protegem as células das injúrias neurotóxicas e podem ser eficazes como um dietético complementar para utilização profilática contra a neurodegeneração progressiva da doença de Parkinson $^{40}$.

Estudos mostraram que os extratos de uva apresentam atividade citotóxica contra células cancerígenas ${ }^{10,22,41-54}$, efeito quimiopreventivo $^{55,56}$, diminuem os efeitos tóxicos de agentes quimioterápicos usados no tratamento de câncer, como idarubicina, 4hidroxiciclofosfamida $^{57}$ e cisplatina $^{58,59}$ e induzem a apoptose celular ${ }^{42,46,51,60,61}$.
Além disso, a atividade antioxidante do extrato de uva merece destaque, pois oferece proteção contra os radicais livres, a peroxidação lipídica ${ }^{9,21,42,52,54,62-69}$ e os danos ao ácido desoxirribonucléico (DNA), inclusive maior do que a oferecida pelas vitaminas $\mathrm{C}, \mathrm{E}$ e betacaroteno $^{57,70-74}$. Estima-se que as procianidinas das uvas possuem potencial antioxidante 20 vezes maior que a vitamina $\mathrm{E}$ e 50 vezes maior que a vitamina $\mathrm{C}^{20,75}$.

As uvas e seus extratos possuem atividade antimutagênica em cepas de Salmonella typhimurium ${ }^{73,76}$, em $\operatorname{ratos}^{49,77}$, em camundongos ${ }^{78-80}$ e em linfócitos humanos ${ }^{81}$.

Além disso, novas pesquisas e trabalhos têm sido desenvolvidos na busca de outras aplicações relacionadas a uva, como: a busca por corantes antociânicos naturais, através de extrações enzimáticas das cascas; a extração, à partir da semente, de óleos ricos em ácidos graxos insaturados, com um elevado teor de ácido linoleico, conhecido também como Ômega 6 (ácido graxo essencial para o homem que não é capaz de sintetizá-lo e que não está presente nos óleos comumente utilizados, como soja, milho e algodão); a utilização desses óleos essenciais por indústrias químicas, cosméticas e farmacêuticas ${ }^{18,82,83}$. 


\section{Conclusão}

O aumento da produção e comercialização da uva e seus derivados e a busca por alimentos com tais características aqui relatadas, tem ampliado o consumo pela população em geral. Dessa forma, a população tem buscado estes inúmeros benefícios já investigados destas frutas, como no tratamento de diversas doenças, neutralização de radicais livres, proteção contra a peroxidação lipídica e danos ao DNA e, a se destacar, suas atividades citotóxicas frente as células tumorais.

Assim sendo, os estudos realizados até o momento são de extrema importância, pois demonstram e elucidam as propriedades desta fruta, assim como incentivam ainda mais o consumo das uvas, seus derivados e até mesmo o reaproveitamento dos seus resíduos. Neste último caso, o uso e extração dos antioxidantes e os compostos de interesse, são aplicados no tratamento de diversas doenças, utilização de corantes naturais e fabricação de produtos químicos, farmacêuticos e cosméticos.

\section{Referências}

1. Mello LMR. Vitivinicultura brasileira: panorama 2017. Bento Gonçalves: Embrapa Informação Tecnológica; 2018.

2. Vieira ACP, Watanabe M, Bruch KL. Perspectiva de Desenvolvimento da Vitivinicultura em Face do Reconhecimento da Indicação de Procedência Vales da Uva Goethe. Revista Gestão, Inovação e Tecnologias. 2012;2(4):327-43.

3. Rombaldi CV, Bergamasqui M, Lucchetta L, Zanuzo M, Silva JA. Produtividade e qualidade de uva, cv. Isabel, em dois sistemas de produção. Revista Brasileira de Fruticultura. 2004;26(1):89-91.

4. Grinder-Pederson L, Rasmussen SE, Bugel S, Jorgensen LV, Dragsted LO, Gubdersen V, Sandström B. Effect od diets based on foods from conventional versus organic production on intake and excretion of flavonoids and markers of antioxidative defense in humans. Jornal of Agriculture and Food Chemistry. 2003;(51):5671-76.

5. Düsman E, Almeida IV, Lucchetta L, Vicentini VEP. Effect of Processing, PostHarvest Irradiation, and Production System on the Cytotoxicity and Mutagenicity of Vitis labrusca L. Juices in HTC Cells. 2014;9(9) Suppl: e107974.

6. Düsman E, Almeida IV, Pinto EP, Lucchetta L, Vicentini VEP. Influence of processing and storage of integral grape juice (Vitis labrusca L.) on its physical and chemical characteristics, cytotoxicity, and mutagenicity in vitro. Genetics and Molecular Research, 2017;16(2) Suppl: gmr16029670.

7. Souza AV, Gomes, GP, Vieira MRS, Vieites RL, Lima GPP. Avaliação de antioxidantes em casca de Vitis sp. Revista Alimentus. 2012;1(2):27-35.

8. Abe LT, Da Motta RV, Lajolo FM, Genovese MI. Compostos fenólicos e capacidade antioxidante de cultivares de uvas Vitis labrusca e Vitis vinifera L. Ciência da Tecnologia de Alimentos. 2007;27(2):394-400. 
9. Nassiri-Asl M, Hosseinzadeh H. Review of the Pharmacological Effects of Vitis vinifera (Grape) and its Bioactive Compounds. Phytotherapy Research. 2009;23(9):1197-1204.

10. Ali K, Maltese F, Choi YH, Verpoorte R. Metabolic constituents of grapevine and grapederived products. Phytochemistry Ver. 2010;9(1):357-78.

11. Guerra CC. Uva para processamento: pós colheita. Brasília: Embrapa Informação Tecnológica; 2003.

12. Mello LMR de. Área de Produção de Uvas: panorama mundial. Jornal da fruta. 2010;18(223):8-9.

13. Rizzon LA, Meneguzzo J. Suco de uva. Brasília: Embrapa Informação Tecnológica; 2007.

14. Rizzon LA, Miele A. Analytical characteristics and discrimination of Brazilian commercial grape juice, nectar, and beverage. Ciência e Tecnologia de Alimentos.2012;32(1):93-7.

15. União Brasileira De Vitivinicultura. Comercialização de Vinhos e Derivados. [acesso em 17 jun. 2013]. Disponível em: http://www.uvibra.com.br/pdf/comercializacao2 008a2013_mar.pdf.

16. SEAB. Secretaria da Agricultura e do Abastecimento do Paraná. [acesso em 21 jul. 2013]. Disponível em: www.seab.pr.gov.br.

17. Sato AJ, Roberto SR. A Viticultura no Paraná. [Acesso em: 21 jul. 2013]. Disponível em:http://www.uepg.br/uepg_departamentos/de fito/htm/labiovegetal/A_Viticultura_No_Parana .pdf.

18. Oliveira DA de. Caracterização Fitoquímica e Biológica de Extratos Obtidos de Bagaço de Uva (Vitis Vinifera) das Variedades Merlot e Syrah [dissertação]. Florianópolis: Universidade Federal de Santa Catarina; 2010.

19. Freitas LS. Desenvolvimento de Procedimentos de Extração do Óleo de Semente de Uva e Caracterização Química dos Compostos Extraídos [tese]. Porto Alegre:
Universidade Federal do Rio Grande do Sul; 2007.

20. Shi J, Yu J, Pohorly JE, Kakuda Y. Polyphenolics in grape seeds-biochemistry and functionality. Journal of Medicinal Food.2003;6(1):291-99.

21. Bagchi DB, Sem CK, Ray SD, Das DK, Bagchi M, Preuss HG, et al. Molecular mechanisms of cardioprotection by a novel grape seed proanthocyanidin extract. Mutation Research-Fundamental and Molecular Mechanisms of Mutagenesis. 2003;523424(1):87-97.

22. Yilmaz Y, Toledo RT. Health aspects of functional grape seed constituents. Trends in Food Science \& Technology. 2004;15(9):42233.

23-Zern TL, Fernandez ML. Cardioprotective effects of dietary polyphenols. Journal of Nutrition. 2005;135(10):2291-94.

24. Lu WD, Qiu J, Zhao GX, Qie LY, Wei XB, Gao HG. Quantitative Mitochondrial Proteomics Study on Protective Mechanism of Grape Seed Proanthocyanidin Extracts Against Ischemia/Reperfusion Heart Injury in Rat. Chemical Research in Chinese Universities. 2012;28(6):1035-40.

25. Alhosin M, Anselm E, Rashid S, Kim JH, Madeira SVF, Bronner C, et al. Redox-Sensitive Up-Regulation of eNOS by Purple Grape Juice in Endothelial Cells: Role of PI3-Kinase/Akt, p38 MAPK, JNK, FoxO1 and FoxO3a. 2013;8(1):1-11.

26. Ammar EM, Said AS, El-Damarawy SL, Suddek GM. Cardioprotective effect of grapeseed proanthocyanidins on doxorubicin-induced cardiac toxicity in rats. Pharmaceutical Biology. 2013;51(3):339-44.

27. Huang LL, Pan C, Wang L, Ding L, Guo K, Wang HZ, et al. Protective effects of grape seed proanthocyanidins on cardiovascular remodeling in DOCA-salt hypertension rats. Journal of Nutritional Biochemistry. 2015;26(1):841-49.

28. Quiñones M, Guerrero L, Suarez M, Pons Z, Aleixandre A, Arola L,et al. Low-molecular 
procyanidin rich grape seed extract exerts antihypertensive effect in males spontaneously hypertensive rats. Food Research International. 2013;51(2):587-95.

29. Stervbo U, Vang O, Bonnesen C. A review of the content of putative chemopreventive phytoalexin resveratrol in red wine. Food Chemistry. 2007;101(1):449-57.

30-Bishayee A, Darvesh AS, Politis T, Mcgory $\mathrm{R}$. Resveratrol and liver disease: from bench to bedside and community. Liver international. 2010;30(8):1103-114.

31. Shin MO, Moon JO. Effect of dietary supplementation of grape skin and seeds on liver fibrosis induced by dimethylnitrosamine in rats. Nutrition Research And Practice. 2010;4(5):369-74.

32. Shin MO, Yoon S, Moon JO. The Proanthocyanidins Inhibit Dimethylnitrosamineinduced Liver Damage in Rats. Archives Of Pharmacal Research. 2010;33(1):167-73.

33. Yalcin E, Oruc E, Cavusoglu K, Yapar K. Protective Effect of Grape Seed Extract on Doxorubicin-Induced Nephrotoxicity ond Hepatotoxicity in Albino Mice. Fresenius Environmental Bulletin. 2012;19(10):2151-58.

34. Ulusoy S, Ozkan G, Yucesan FB, Ersöz S, Orem A, Alkanat M, et al. Anti-apoptotic and anti-oxidant effects of grape seed proanthocyanidin extract in preventing cyclosporine A-induced nephropathy. Nephrology. 2012;17(4):372-79.

35. Charradi K, Elkahoui S, Karkouch I, Limam F, Hamdaoui G, Hassine FB, et al. Grape seed and skin extract alleviates high-fat diet-induced renal lipotoxicity and prevents copper depletion in rat. Applied Physiology, Nutrition and Metabolism. 2013;38(3):259-67.

36. Chis IC, Ungureanu MI, Marton A, Simedrea R, Muresan A, Postescu ID,et al. Antioxidant effects of a grape seed extract in a rat model of diabetes mellitus. Diabetes \& Vascular Disease Research. 2009;6(3):200-4.

37. Hou WR, Hou YL, Wu GF, Song Y, Su XL, Sun B, et al. cDNA, genomic sequence cloning and overexpression of ribosomal protein gene $\mathrm{L} 9$
(rpL9) of the giant panda (Ailuropoda melanoleuca). Genet Mol Res. 2011;10(3):157688.

38. Liu MY, Yin Y, Ye XY, Zeng M, Zhao Q, Keefe DL, et al. Resveratrol protects against ageassociated infertility in mice. Human Reproduction. 2013;28(3):707-17.

39. Wang J, Ho L, Zhao W, Ono K, Rosensweig $\mathrm{C}$, Chen L, et al. Grape-derived polyphenolics prevent $\mathrm{A} \beta$ oligomerization and attenuate cognitive deterioration in a mouse model of Alzheimer's disease. Journal of Neuroscience. 2008;28(5):6388-92.

40. Zhang Y, Hu XS, Chen F, Wu JH, Liao XJ, Wang ZF. Stability and color characteristics of PEF treated cyaniding-3-glicoside during storage. Food Chem. 2008:106(1):669-79.

41. Babich H, Reisbaum AG, Zuckerbraun HL. In vitro response of human gingival epithelial $S$ $\mathrm{G}$ cells to resveratrol. Toxicology Letters. 2000;114(1-3):143-53.

42. Bagchi D, Bagchi M, Stohs SJ, Das DK, Ray $\mathrm{SD}$, Kuszynski CA, et al. Free radicals and grape seed proanthocyanidin extract: importance in human health and disease prevention. Toxicology. 2000;148(2-3):187-97.

43. Nakagawa H, Kiyozuka Y, Uemura Y, Senzaki H, Shikata N, Hioki K, et al. Resveratrol inhibits human breast cancer cell growth and may mitigate the effect of linoleic acid, a potent breast cancer cell stimulator. Journal of Cancer Research and Clinical Oncology. 2001;127(4):258-64.

44. Sharma G, Tyagi AK, Singh RP, Chan DCF, Agarwal R. Synergistic anti-cancer effects of grape seed extract and conventional cytotoxic agent doxorubicin against human breast carcinoma cells. Breast Cancer Research and Treatment. 2004;85(1):1-12

45. Singh RP, Tyagi AK, Dhanalakshmi S, Agarwal R, Agarwal C. Grape seed extract inhibits advanced human prostate tumor growth and angiogenesis and pregulates insulin-like growth factor binding protein-3. International Journal of Cancer. 2004;108(1):733-40. 
46. Zhang XY, Bai DC, Wu YJ, Li WG, Liu NF. Proanthocyanidin from grape seeds enhances anti-tumor effect of doxorubicin both in vitro and in vivo. Pharmazie. 2005;60(7):533-8.

47. Zhang XY, Li WG, Wu YH, Bai DC, Liu NF. Proanthocyanidin from grape seeds enhances doxorubicin-induced antitumor effect and reverses drug resistance in doxorubicin-resistant K562/DOX cells. Canadian Journal of Physiology and Pharmacology. 2005;83(3):30918.

48. Zhang XY, Li WG, Wu YH, Zheng TZ, Li W, Qu SY, et al. Proanthocyanidin from grape seeds potentiates anti-tumor activity of doxorubicin via immunomodulatory mechanism. International Immunopharmacology. 2005;5(7-8):1247-57.

49. Jung K, Wallig MA, Singletary KW. Purple grape juice inhibits 7, 12-dimethylbenz[a] anthrancene (dmba)-induced rat mammary tumorigenesis and in vivo dmba-dna adduct formation. Cancer letters. 2006;233(2):279-88.

50. Zheng M, Chen RF, Zhong HY, Lin QY, Wang XQ, Zhao ZW, et al. Side-effects of resveratrol in HepG2 cells: Reduced pten and increased bcl-xl mRNA expression. Molecular Medicine Reports. 2012;6(6):1367-70.

51. Zhang MH, Zhou X, Zhou KY. Resveratrol inhibits human nasopharyngeal carcinoma cell growth via blocking pAkt/p70S6K signaling pathways. International Journal of Molecular Medicine.2013;31(3):621-7.

52. Nowshehri JA, Bhat ZA, Shah MY. Blessings in disguise: bio-functional benefits of grape seed extracts. Food Research International. 2015;77(3):333-48.

53. Rocha AM, Düsman E, Dalmolin, IAL. Produção de extratos dos resíduos de indústria vinícola e sua avaliação antioxidante e citotóxica [trabalho de conclusão de curso]. Francisco Beltrão: Universidade Tecnológica Federal do Paraná; 2018.

54. Lopes NB, Almeida IV, Lucchetta L, Düsman E, Vicentini VEP. Cytotoxic effects of Vitis labrusca (fox grape) whole juices on human tumor and non-tumor cells, in vitro. Genetics and
Molecular Research, 2019;18(2) Suppl: gmr18236.

55. Singletary KW, Jung KJ, Giusti $M$. Anthocyanin.rich grape extract blocks breast cell DNA damage. Journal of Medicinal Food. 2007;10(2):244-51.

56. Song XY, Siriwardhana N, Rathore K, Lin DG, Wang HCR. Grape Seed Proanthocyanidin Suppression of Breast Cell Carcinogenesis Induced by Chronic Exposure to Combined 4(Methylnitrosamino)-1-(3-Pyridyl)-1-Butanone and Benzo[a]Pyrene. Molecular Carcinogenesis, 2010;49(1):450-63.

57. Joshi SS, Kuszynski CA, Bagchi M, Bagchi D. Chemopreventive effects of grape seed proanthocyanidin extract on chang liver cells. Toxicology. 2000;155(1-3):83-90.

58. Saad AA, Youssef MI, El-Shennawy LK. Cisplatin induced damage in kidney genomic DNA and nephrotoxicity in male rats: the protective effect of grape seed proanthocyanidin extract. Food and Chemical Toxicology. 2009;47(7):1499-1506.

59. Cetin A, Arslanbas U, Saraymen B, Canoz O, Ozturk A, Sagdic O. Effects of grape seed extract and Origanum onites essential oil on Cisplatin-induced hepatotoxicity in rats. UHOD - Uluslararasi Hematoloji-Onkoloji Dergisi. 2011; 21(3):133-40.

60. Soleas GJ, Goldberg DM, Grass L, Levesque M, Diamandis EP. Do wine polyphenols modulate p53 gene expression in human cancer lines? Clinical Biochemistry. 2001;34(5):41520.

61. Tyagi A, Agarwal R, Agarwal C. Grape seed extract inhibits EGF-induced and constitutively active mitogenic signaling but activates JNK in human prostate carcinoma DU145 cells: possible role in antiproliferation and apoptosis. Oncogene. 2003;22(9):1302-16.

62. Ray SD, Lam TS, Rotollo JA, Phadke S, Patel C, Dontabhaktuni A, et al. Oxidative stress is the master operator of drug and chemicallyinduced programmed and unprogrammed cell death: Implications of natural antioxidants in vivo. Biofactors. 2004:21(1-4):223-32. 
63. Adriana M, Suciu S, Clichici S, Daicoviciu D, Pop N, Potescu ID. Study on the effects of grape seed extract in ehrlich ascitic carcinoma. Bulletin Of The University Of Agricultural Sciences And Veterinary Medicine. 2006;63(1. 2):114-19.

64. Baydar NG, Özkan G, Yasar S. Evaluation of the antiradical and antioxidant potential of grape extracts. Food Control. 2007:18(9):113136.

65. Çetin A, Kaynar L, Koçyiğit I, Hacioğlu SK, Saraymen R, Öztürk A, et al. The effect of grape seed extract on radiation-induced oxidative stress in the rat liver. Turkish Journal of Gastroenterology. 2008;19(2):92-8.

66. Pieniz S, Colpo E, Oliveira VR, Estefanel V, Andreazza R. Avaliação In Vitro do Potencial Antioxidante de Frutas e Hortaliças. Ciência e Agrotecnologia. 2009;33(2):552-9.

67. Pinheiro FV, Pimentel VC, De Bona KS, Scola G.; Salvador, M.; Funchal, C.; Moretto, M.B. Decrease of adenosine deaminase activity and increase of the lipid peroxidation after acute methotrexate treatment in young rats: Protective effects of grape seed extract. Cell Biochemistry and Function. v. 28, n. 1, p. 89-94, 2010.

68. Fernandes F, Ramalhosa E, Pires P, Verdial J, Valentão P, Andrade P, et al. Vitis vinífera leaves towards bioactivity. Industrial Crops and Products. 2013;43(1):434-40.

69. Maestre R, Douglass JD, Kodukula S, Medina I, Storch J. Alterations in the Intestinal Assimilation of Oxidized PUFAs Are Ameliorated by a Polyphenol-Rich Grape Seed Extract in an In Vitro Model and Caco-2 Cells. Journal of Nutrition. 2013;143(3):295-301.

70. Edenharder R, Sager JW, Glatt H, Muckel E, Platt KL. Protection by beverages, fruits, vegetables, herbs, and flavonoids against genotoxicity of 2-acetylaminofluorene and 2amino-1-methyl-6-phenylimidazo[4,5-

b]pyridine (phip) in metabolically competent v79 cells. Mutation research. 2002;521(1-2):5772.

71. Ohsawa KI, Nakagawa SY, Kimura M, Shimada C, Tsuda S, Kabasawa K, Kawaguchi S, Sasaki YF. Detection of in vivo genotoxicity of endogenously formed n-nitroso compounds and suppression by ascorbic acid, teas and fruit juices. Mutation research. 2003;539(1-2):65-76.

72. Park YK, Park E, Kim JS, Kang MH. Daily grape juice consumption reduces oxidative DNA damage and plasma free radical levels in healthy Koreans. Mutation Research. 2003;529(1-2):7786.

73. Stagos D, Kazantzoglou G, Theofanidou D, Kakalopoulou G, Magiatis P, Mitaku S, Kouretas D. Acitivity of grape extracts from Greek varieties of Vitis vinifera against mutagenicity induced by bleomycin and hydrogen peroxide in Salmonelle typhimurium strain TA 102. Mutation Research. 2006;609(2):165-75.

74. Rezende AAA, Graf U, Guterres ZR, Kerr WE, Spanó MA. Protective efects of proanthocyanidins of grape (Vitis vinifera L.) seeds DNA damage induced by Doxorubicin in somatic cells of Drosophila melanogaster. Food and Chemical Toxicology. 2009;47(7):1466-72.

75. Bagchi D, Bagchi M, Stohs SJ, Ray SD, Sen CK, Preuss HG. Cellular protection with proanthocyanidins derived from grape seeds. Alcohol and Wine in Health and Disease. 2002;957(1):260-70.

76. Catterall F, Souquet JM, Cheynier V, Clifford MN, Ioannides C. Modulation of the mutagenicity of food carcinogens by oligomeric and polymeric procyanidins isolated from grape seeds: synergistic genotoxicity with $\mathrm{N}$ nitrosopyrrolidine. Journal of the Science of Food and Agriculture. 2000;80(1):90-101.

77. Attia SM, Bakheet AS, Al-Rasheed NM. Proanthocyanidins produce significant attenuation of doxorubicin-induced mutagenicity via suppression of oxidative stress. Oxidative Medicine and Cellular Longevity. 2010;3(6):404-13.

78. Bagchi D, Ray SD, Patel D, Bagchi M. Protection against drug- and chemical-induced multiorgan toxicity by a novel IH636 grape seed proanthocyanidin extract. Drugs under Experimental and Clinical Research. 2001;27(1):3-15. 
79. Yalcin E, Oruc E, Cavusoglu K, Yapar K. Protective Role of Grape Seed Extract Against Doxorubicin-Induced Cardiotoxicity and Genotoxicity in Albino Mice. Journal of Medicinal Food. 2010;13(4):917-25.

80. Abd El Kader MA, El-Sammad N M, Fyiad A. Effect of Grape Seeds Extract in the Modulation of Matrix Metalloproteinase-9 Activity and Oxidative Stress Induced By Doxorubicin in Mice. Life Science Journal-Acta Zhengzhou University Overseas Edition. 2011;8(3):510-15.

81. Szeto YT, Lee KY, Kalle W, Pak, SC. Protective effect of grape seed extracts on human lymphocytes: A preliminary study. Applied Physiology, Nutrition and Metabolism. 2013;38(3):275-79.

82. Montibeller MJ. Extração enzimática em cascas de uva: processo sustentável para obtenção de corante antociânico [dissertação]. Porto Alegre: Universidade Federal do Rio Grande do Sul; 2017.

83. Souza EXN de S. Desenvolvimento e avaliação de lipossomas com bioativos de uva (Vitis vinifera) para medicamentos e cosméticos [dissertação]. Salvador: Universidade Federal da Bahia; 2015.

Data de submissão: 27/11/2019

Data de aprovação:13/12/2019 